\title{
Toxoplasmose: assistência pré-natal sob a abordagem da rede cegonha em Itaparica-Bahia-Brasil entre 2013 a 2016
}

\author{
Toxoplasmosis: prenatal care under the "rede cegonha" strategy in Itaparica-Bahia-Brazil \\ from 2013 to 2016
}

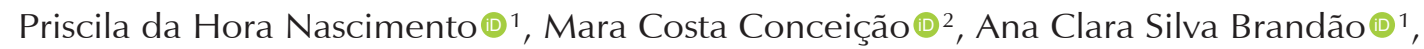
Pedro Paulo Oliveira Carneiro ${ }^{1}{ }^{1}$, Rúbia Suely Santana Costa $\mathbb{1}^{1}$

${ }^{1}$ Centro Universitário Maurício de Nassau, Salvador, BA.

2Universidade do Estado da Bahia (UNEB), Salvador, BA.

RESUMO

Introdução: A toxoplasmose é uma doença infecciosa de grande importância clínica para gestantes devido à possibilidade de transmissão congênita. A rede cegonha é uma estratégia utilizada para assistência ao pré-natal inserida na atenção básica à saúde, tem finalidade de reduzir índices de mortalidade materna e neonatal.

Objetivo: Estabelecer a relação entre o processo de diagnóstico sorológico para toxoplasmose em gestantes e a atuação da rede cegonha.

Materiais e Métodos: Trata-se de um estudo de caráter descritivo retrospectivo de base documental, constituído por 461 prontuários de gestantes atendidas no período de 2013 a 2016 no município de Itaparica/BA. Abordam dados clínicos e epidemiológicos das gestantes inseridas na rede cegonha e vinculadas a uma das unidades de saúde da família de Itaparica/BA.

Resultados: Foram identificadas $302(65,5 \%)$ gestantes que realizaram sorologia para toxoplasmose, sendo que 3,65\% foram susceptíveis, 11,6\% imunes, 1,3\% com possível infecção aguda e 83,45\% tiveram perfil clínico inconcluso para toxoplasmose.

Conclusão: A rede cegonha facilita o acesso ao acompanhamento pré-natal e diagnóstico sorológico. No entanto, há dificuldades no que se refere à realização de exames que auxiliem a conclusão diagnóstica.

Palavras-chave: sorologia; imunoglobulina G; imunoglobulina M; saúde da família; rede cegonha.

Introduction: Toxoplasmosis is an infectious disease of great clinical importance for pregnant women due to the possibility of congenital transmission. The "rede cegonha" is a strategy used for prenatal care inserted in the basic health care system, aiming to reduce rates of maternal and neonatal mortality.

Objective: To establish the relationship between the serological diagnosis process for toxoplasmosis in pregnant women and the performance of "rede cegonha".

Materials and Methods: This is retrospective descriptive study with a documental basis, consisting of 461 records of pregnant women assisted in the city of Itaparica/BA, Brazil, from 2013 to 2016. The research shows clinical and epidemiological data of pregnant women inserted in the "rede cegonha", which is related to one of the family health units of Itaparica.

Results: 302 (65.5\%) pregnant women who underwent serology for toxoplasmosis were identified, with 3.65\% considered as susceptible, $11.6 \%$ immune, $1.3 \%$ with a possible acute infection and $83.45 \%$ with an inconclusive clinical profile for toxoplasmosis. Conclusion: The "rede cegonha" strategy facilitates access to prenatal monitoring and serological diagnosis. However, there are difficulties regarding the performance of medical exams that help finding a diagnostic conclusion.

Keywords: serology; immunoglobulin G; immunoglobulin M; family health; rede cegonha. 


\section{INTRODUÇÃO}

A infecção causada pelo Toxoplasma gondii durante a gravidez é uma das principais causas da infecção congênita grave $^{1,2}$. O parasita atinge o feto através da placenta ou devido à reativação de cistos teciduais em gestantes imunodeprimidas, e pode resultar em aborto, doenças sistêmicas ou danos neuro-oftalmológicos ${ }^{1,3-5}$.

Apesar de várias pesquisas enfatizarem a infecção de gestantes pelo $T$. gondii, há muitos problemas não resolvidos na toxoplasmose congênita e que precisam ser abordados, a exemplo da assistência da triagem pré-natal, a variabilidade da resposta sorológica materna e sua relação custo-eficácia ${ }^{6}$. Estudos apontam que apesar do perfil antiparasitário materno e tratamento pós-natal a abordagem mais eficaz para o controle da doença é o uso de estratégias médicas preventivas, especialmente programas de acompanhamento materno durante a gravidez e em longo prazo para monitorar o prognóstico ocular e neurológico da criança ${ }^{7,8}$.

Nesta perspectiva, a integralidade das ações de Assistência à Saúde Pública garante uma nova intervenção visando à família como centro para o atendimento 9 . Esse atendimento quando efetivo permite que as informações sobre a prevenção da doença sejam alcançadas pela população. Uma estratégia de cuidados para a saúde da mulher desde o planejamento familiar ao puerpério é a rede cegonha, programa criado pelo Governo Federal para garantir o direito e a atenção à mulher durante o período gestacional, parto, puerpério, e crianças de até dois anos de idade. Proporciona exames como sorologias para toxoplasmose, rubéola, citomegalovírus, sífilis, Human Immunodeficiency Virus (HIV), doença de Chagas, hepatites B e C, Human T Lymphotropic Vírus (HTLV), hipotireoidismo, anemia falciforme e outras hemoglobinopatias ${ }^{9,10}$.

A imunidade humoral é a principal resposta para identificação e proteção da gestante contra a toxoplasmose. A importância de anticorpos humanos da classe lgM, IgA, IgE e IgG2 específicos para T. gondii tornou-se uma poderosa ferramenta imunológica para distinguir os indivíduos recéminfectados daqueles na fase crônica da infecção ${ }^{11,12}$, o teste de avidez soma-se ao diagnóstico para detectar a soroconversão recente a partir dos títulos de $\lg \mathrm{G}^{13,14}$. Quando identificado precocemente estes pacientes são encaminhados para tratamento diminuindo a possibilidade de transmissão via transplacentária 5 .

Independentemente da espécie do $T$. gondii, a IgM aparece no soro de 3 a 10 dias após o contato com o protozoário, apesar de serem específicas da fase aguda, podem ser encontradas anos depois da infecção, provavelmente devido a reativação ou até mesmo pela persistência do parasita ${ }^{15}$. Assim, a consulta durante o pré- natal se torna a principal forma de prevenção primária para a toxoplasmose congênita ${ }^{4}$, porque o risco de transmissão para o feto está associado à idade gestacional, quanto mais cedo o contato com o parasito, maior o risco de transmissão ${ }^{16}$. O objetivo deste estudo foi estabelecer a relação entre o diagnóstico sorológico para toxoplasmose em gestantes e avaliar a funcionalidade da rede cegonha na triagem prénatal.

\section{MATERIAIS E MÉTODOS}

Trata-se de um estudo de caráter descritivo retrospectivo, de base documental. Foram analisados 461 prontuários de gestantes inseridas no programa de triagem pré-natal da rede cegonha, integrada às Unidades de Saúde da Família (USF) no município de Itaparica - Bahia.

Os dados foram coletados a partir dos prontuários das nove Unidades de Saúde da Família de Itaparica, sendo eles: Centro, Alto das Pombas, Ponta de Areia, Amoreiras, Manguinhos, Porto dos Santos, Marcelino, Mocambo e Misericórdia. Os critérios para a seleção dos prontuários foram baseados na apresentação dos exames $\beta$-HCG ou ultrassonografia que confirmaram a gestação, também foram selecionados os prontuários de gestantes que além da triagem da rede cegonha utilizaram diagnóstico laboratorial particular que serviram para comparação de resultados. Durante a pesquisa foram registradas a idade gestacional, data de realização da primeira consulta e resultados sorológicos trimestrais para toxoplasmose durante o acompanhamento clínico, em seguida, observou-se se a realização de exames confirmatórios (repetição de lgM, IgG e teste de avidez para IgG).

As gestantes foram classificadas em imunes (IgM não reagente e IgG reagente), possível infecção aguda (IgM reagente ou indeterminado com realização de $\lg \mathrm{ou}$ não), susceptíveis (IgM e IgG não reagentes) e inconclusas (realizaram apenas $\operatorname{lgM}$ que apresentou resultado não reagente ou indeterminado, neste caso não há como discernir se a gestante é susceptível à infecção ou se está cronicamente infectada por $T$. gondii).

A triagem sorológica da rede cegonha é realizada por teste em papel filtro por meio do método de Enzyme Linked Immuno Sorbent Assay (ELISA), sem resultados quantitativos, enquanto exames realizados à parte foram registrados para demonstrar a titulação dos anticorpos.

Os dados são apresentados em média e desvio padrão, o teste não paramétrico de Mann-Whitney foi usado para comparar os grupos de gestantes de acordo com a idade. O presente estudo foi aprovado pelo Comitê de Ética em Pesquisa Instituto Mantenedor de Ensino Superior (IMES) localizado na Faculdade de Tecnologia e Ciências em Salvador/BA, parecer de no 1.773 .236 . 


\section{RESULTADOS}

No período de junho de 2013 a setembro de 2016 foram encontrados 461 prontuários de gestantes inseridas no programa de triagem pré-natal da rede cegonha. Destes, 125 (27\%) corresponderam ao período de junho de 2013 a maio de 2014, 164 (35\%) em junho de 2014 a maio de 2015 e 172 (38\%) de junho de 2015 a setembro de 2016.

De todas variáveis analisadas, foi identificada 2.446 consultas, realizados 248 partos, relatados 104 casos de abortos. Destes prontuários, 157 grávidas apresentaram idade entre 13 a 20 anos e 304 de 21 a 41 anos (Tabela 1). Sendo que as gestantes acima de 30 anos realizaram a primeira consulta três semanas antes do que as mais novas e também tiveram mais filhos e ocorrências de abortos. Quanto à sorologia, 159 mulheres foram atendidas, mas não fizeram o teste para toxoplasmose. Assim, o perfil sorológico foi determinado para 302 grávidas (Figura 1).
Após identificação do perfil sorológico, fez-se uma análise do acompanhamento pré-natal destas gestantes. Foi observado que somente quatro iniciaram as consultas nos primeiros meses da gestação e nenhuma realizou sorologia para IgM e IgG anti-T.gondii em todos os trimestres de acompanhamento (Tabela 2).

Tabela 1. Características clínicas e epidemiológicas das gestantes que receberam atendimento pré-natal pelo programa da rede cegonha em Itaparica/BA.

\begin{tabular}{lcc|}
\hline Variáveis das gestantes & & Valor de $\boldsymbol{p}$ \\
\hline Idade (anos) & $24 \pm 6^{*}$ & $<0,05^{*}$ \\
Idade Gestacional (Semanas) & $13 \pm 7^{*}$ & $<0,05^{*}$ \\
Número de Gestações & $748^{* *}(1,8 \pm 1,2)$ & $>0,05^{*}$ \\
Número de Partos & $240^{* *}(0,5 \pm 0,9)$ & $>0,05^{*}$ \\
Número de Abortos & $104^{* *}(0,2 \pm 0,6)$ & $>0,05^{*}$ \\
Número de Consultas & $2,446^{* *}(5 \pm 2)$ & $>0,05^{*}$ \\
\hline "Média e desvio padrão. & &
\end{tabular}

* Significância estatística $p<0,05$. Teste não paramétrico de Mann-Whitney. ** Valores absolutos.

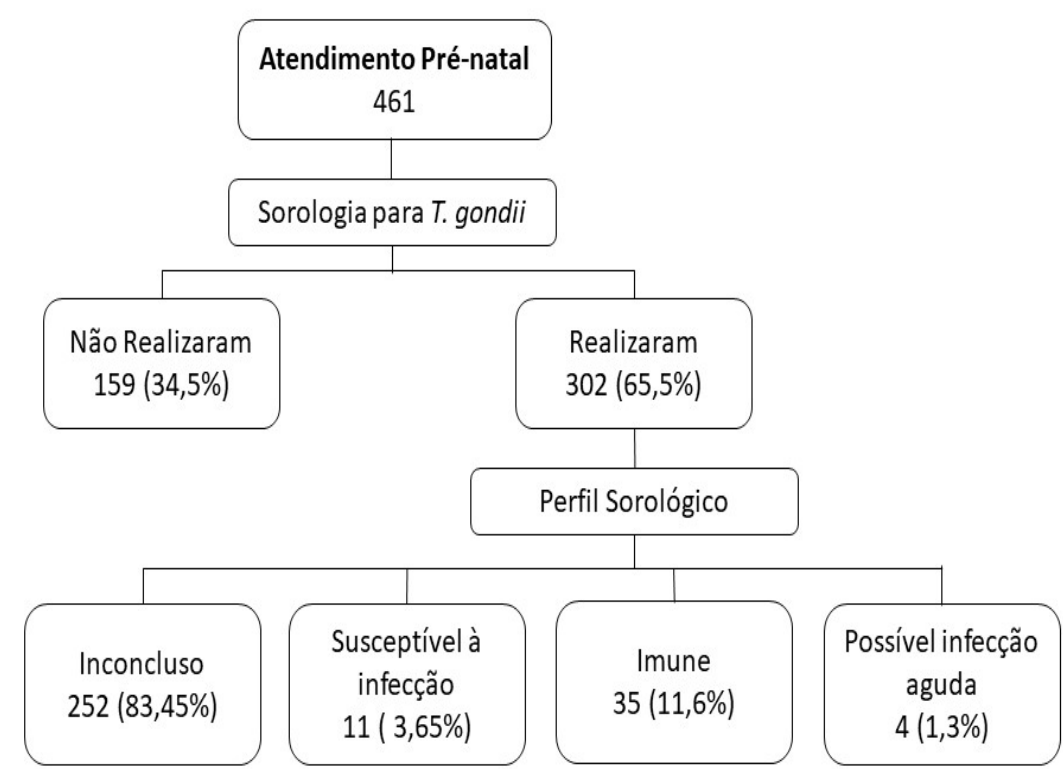

Figura 1. Estratificação das gestantes assistidas pela rede cegonha de acordo o diagnóstico sorológico.

Tabela 2. Acompanhamento sorológico das gestantes que apresentaram IgM anti-Toxoplasma gondii.

\begin{tabular}{|c|c|c|c|c|c|c|}
\hline \multirow{2}{*}{ Gestante } & \multicolumn{2}{|c|}{10 Trimestre } & \multicolumn{2}{|c|}{ 20 Trimestre } & \multicolumn{2}{|c|}{30 Trimestre } \\
\hline & $\lg M$ & IgG & IgM & IgG & IgM & IgG \\
\hline 1 & IND & Reagente & $\begin{array}{c}\text { IND } \\
(1,02 \text { UI })\end{array}$ & $\begin{array}{c}\text { Reagente } \\
\text { (650 UI) }\end{array}$ & NR & - \\
\hline 2 & IND & - & NR & $\begin{array}{c}\text { Reagente } \\
(2,7 \text { UI })\end{array}$ & - & - \\
\hline 3 & Reagente & - & $\begin{array}{c}\text { Reagente } \\
(4,2 \cup \mathrm{UI})\end{array}$ & $\begin{array}{c}\text { Reagente } \\
(1,2 \cup I)\end{array}$ & NR & - \\
\hline 4 & Reagente & $N R$ & - & - & - & - \\
\hline
\end{tabular}

IND: Indeterminado; NR: Não reagente; -: Não realizou o exame; UI: Unidades internacionais; IgM: Imunoglobulina M; IgG: Imunoglobulina G. A triagem sorológica é realizada por teste em papel filtro pelo método de ELISA. 


\section{DISCUSSÃO}

Um dos parâmetros importantes para consolidação dos programas de saúde está relacionado à oferta dos serviços pelas unidades de saúde, principalmente na atenção básica, e a demanda dos usuários ${ }^{17}$. Na realização da consulta prénatal deve ser feito a investigação da história pregressa da gestante como uma das habilidades básicas e necessárias instituídas pelos Programas de Assistência à Saúde ${ }^{18}$. Apesar de todas 461 gestantes terem feito anamnese na primeira consulta, foram encontradas lacunas no preenchimento de vários dados referentes às informações epidemiológicas e de acompanhamento para os casos de gravidez de risco.

Neste estudo, foi considerada a idade como uma variável relevante em relação ao acompanhamento médico gestacional, e percebeu-se que mulheres mais velhas apresentaram maior número de gestações, partos, ocorrência de abortos e também são mais comprometidas com a continuidade do pré-natal. De acordo com O'Connor et al. ${ }^{19}$, as mulheres com média de 30 anos têm preocupações adicionais sobre os ricos de o feto desenvolver anomalias e procuram atendimento durante a gestação.

Outro resultado encontrado foi relacionado à interrupção das consultas. Vários fatores podem ter contribuído para a desistência, a exemplo da falta de conhecimento dos riscos em não realizar o pré-natal, a dificuldade do acesso ao sistema de saúde, distância de sua moradia para as localidades dos postos de saúde, a inoperância do atendimento, problemas emocionais e desistência da gravidez, no entanto os prontuários não apresentaram dados suficientes para o embasamento dessa realidade. Segundo Lima et al. ${ }^{8}$ os dados da literatura mostram que dificuldades como, trabalhos, filhos, distância, atividades do lar são desafios enfrentados rotineiramente pelas gestantes.

A necessidade da realização de sorologia para toxoplasmose na gestação reside no fato de ser a melhor forma de diagnóstico da infecção possibilitando assim o tratamento precoce para inibir a transmissão congênita do Toxoplasma gondii ${ }^{4}$. Este trabalho mostra que 159 gestantes foram inseridas no programa, mas não fizeram a sorologia durante as consultas. No entanto, a efetividade dos programas de saúde materno-infantil não está exclusivamente vinculada à ação dos profissionais, mas também está condicionada ao nível de estruturação e de seus derivados como orientação familiar, comunitária e competência cultural ${ }^{20}$.

Os testes que apresentaram perfil clínico inconcluso podem ser procedentes do fato que a rede cegonha apenas disponibiliza sorologia para IgM, visto que não se discerne a gestante imune da susceptível à infecção pelo Toxoplasma gondii1 ${ }^{17}$. É necessário a avaliação de $\lg M$ e $\lg G$ anti-T.gondii para definição do perfil sorológico, pois resultado de lgM não deve ser analisado de forma independente para determinar a fase da infecção ${ }^{21}$. Referente ao diagnóstico que compreende susceptibilidade à infecção é indispensável fazer a cada consulta para garantir o conhecimento da infecção adquirida durante a gravidez ${ }^{4}$, contudo as repetições mensais de sorologia não foram encontradas nos prontuários e não tem dados suficientes que possam assegurar a realização do aconselhamento.

Não foi encontrado registro de encaminhamento para uma maternidade como gravidez de alto risco para as quatro gestantes que apresentaram diagnóstico com possível infecção aguda, IgM reagente ou indeterminada, também não foi realizado exames confirmatórios como sorologia para IgG e nem o teste de avidez de IgG. O teste de avidez de IgG consiste em uma ferramenta padrão que viabiliza o diagnóstico do tempo estimado da infecção pelo $T$. gondii, possibilitando a distinção de infecção aguda ou crônica ${ }^{22}$. É prudente que tal teste seja feito preferivelmente com a mesma amostra para excluir resultados falsos-positivos para $\lg \mathrm{M}^{23}$. A liberação de resultados falso-positivos pode ocasionar consideráveis complicações, como à realização da amniocentese desnecessária, que pode causar até aborto ${ }^{24,17}$.

A rede cegonha só disponibiliza o teste imunoenzimático para IgM anti-T.gondii, isso pode ser um fator limitante no serviço, pois não identifica o perfil sorológico das gestantes; a dificuldade da interpretação dos laudos e pouco conhecimento sobre os agravos da doença pelos profissionais envolvidos na triagem é outro fator a ser discutido, haja vista que muitos prontuários apresentaram resultados inconclusos sem repetição dos diagnósticos com resultado indeterminado. Segundo o Ministério da Saúde, as pacientes com possível diagnóstico de infecção aguda necessitam ser encaminhadas para confirmação do diagnóstico e, se necessário, para o tratamento devido ao risco acentuado de exposição do concepto $^{25,26}$. Para Cantos e colaboradores é comum haver dificuldades na interpretação dos resultados sorológicos voltados à toxoplasmose gestacional. Contudo, nos casos de infecção aguda confirmada são indispensáveis medidas terapêuticas imediatas ${ }^{4,23,27,28}$. Apesar das pacientes serem tratadas em hospitais de referência, compete a Estratégia de Saúde da Família (ESF) continuar o acompanhamento visando prestação de cuidados à gestante e família ${ }^{26}$.

A ausência de testes complementares pode ter ocasionado erros básicos na interpretação do resultado, pois as gestantes IgM positiva ou resultado indeterminado com $\operatorname{lgG}$ reagente, sem a realização do teste de avidez de $\lg G$ não confere ciência de imunidade para toxoplasmose, embora a repetição da sorologia nos trimestres subsequentes tenha demonstrado soroconversão ${ }^{22}$. Todavia IgG positivo com 
ausência do diagnóstico para IgM não infere segurança de imunidade, portanto baixa avidez para IgG pode determinar a infecção aguda ainda em curso ${ }^{28}$. Uma forma de aprimorar o diagnóstico de triagem sorológica, que poderia ser implementado pela rede cegonha, seria a liberação dos títulos sorológicos de IgM e IgG nos laudos, para auxiliar na caracterização do grau de infecção para toxoplasmose e compreender melhor a soroconversão $0^{1,17,28}$.

A partir da análise dos dados obtidos através das unidades de saúde, percebeu-se fatores limitantes na triagem e no diagnóstico para toxoplasmose gestacional, isso inclui início tardio por parte de algumas gestantes, demora da entrega de resultados e retorno da paciente à ESF onde iniciou o pré-natal. A migração das gestantes para a outra ESF sem informar sobre o atendimento anterior é uma variável que prejudica o rastreamento da infecção por não haver um sistema integrado entre as unidades. Demonstrou-se também maior prevalência de gestantes com diagnóstico inconcluso para toxoplasmose, além das dificuldades reais no tempo de liberação dos exames. A rede cegonha como Programa de Proteção à Gestante facilita o acesso ao diagnóstico sorológico e ao acompanhamento durante o pré-natal, entretanto não fornece a sorologia para $\operatorname{lgG}$ e nem a avidez para IgG que auxiliaria na conclusão diagnóstica.

\section{AGRADECIMENTOS}

Agradecemos a equipe de Enfermagem e a Secretaria Municipal de Saúde pela acessibilidade e esclarecimentos sobre a rede cegonha do Município de Itaparica/BA.

\section{REFERÊNCIAS}

1. Reis MM, Tessaro MM, D'Azevedo PA. Perfil sorológico para toxoplasmose em gestantes de um hospital público de Porto Alegre. Rev. Bras. Ginec. Obstet [periódico online]. 2006; 28(3):158-164. Disponível em: https://doi.org/10.1590/S010072032006000300004

2. Mccabe RE, Remington JS. Toxoplasma gondii. In: Mandell GL, Douglas RG, Bennett JE, editors. Principles and Practice of Infectious Diseases. Churchill Livingstone Inc.; New York: 1990.

3. Bittencourt LH, Lopes-Mori FM, Mitsuka-Breganó R, ValentimZabott M, Freire RL, Pinto SB, Navarro IT. Soroepidemiologia da toxoplasmose em gestantes a partir da implantação do programa de vigilância da toxoplasmose adquirida e congênita em municípios da região oeste do Paraná. Rev. Bras. Ginec. Obstet. [periódico online]. 2012; 34:63-68. Disponível em: http://www. scielo.br/pdf/rbgo/v34n2/a04v34n2.pdf

4. Lopes-Mori FMR, Mitsuka-Breganó R, Capobiango JD, Inoue IT, Reiche EMV, Morimoto HK, Casella AMB, Bittencourt LHFB, Freire RL, Navarro IT. Programa de controle da toxoplasmose congênita. Rev. Assoc. Med. Bras. [periódico online]. 2011; 57(5):594-599. Disponível em: https://doi.org/10.1590/S010442302011000500021
5. Montoya JG, Remington JS. Management of Toxoplasma gondii infection during pregnancy. Clin. Infect. Dis. [periódico online]. 2008; 47(4):554-66. Disponível em: https://www.ncbi.nlm.nih. gov/pubmed/18624630. https://doi.org/10.1086/590149

6. Porto AMF, Amorim MMR, Coelho ICN, Santos LC. Perfil sorológico para toxoplasmose em gestantes atendidas em maternidade. Rev. Assoc. Med. Bras. [periódico online]. 2008; 54(3):242-248. Disponível em: https://doi.org/10.1590/S010442302008000300018

7. Fonseca AL, Silva RA, Fux B, Madureira AP, Sousa FF, Margonari C. Aspectos epidemiológicos da toxoplasmose e avaliação da sua prevalência em mulheres grávidas. Soc. Bras. Med. Trop. [periódico online]. 2012; 45(3):1-11. Disponível em: https://doi. org/10.1590/S0037-86822012000300015

8. Lima AF, Melo AMAA, Ferreira MA. Pré-natal: um desafio para as gestantes acompanhadas nas unidades de saúde da família no município de Serra Talhada - PE. Saúde Coletiva em Debate. 2012; 2 (1):31-40.

9. Ministério da Saúde (Brasil). Portaria no 1.459, de 24 de junho de 2011. Institui, no âmbito do Sistema Único de Saúde - SUS - a Rede Cegonha. Diário Oficial [da] República Federativa do Brasil. 2011. Disponível em: http://bvsms.saude.gov.br/bvs/saudelegis/ gm/2011/prt1459_24_06_2011.html

10. Instituto de Pesquisa e Ensino Diagnóstico IPED APAE. Unidade de Triagem Pré-natal [Internet]. 2016. Disponível em: http://www. ipedapae.org.br/triagem-pre-natal

11. Sensini A. Toxoplasma gondii infection in pregnancy: opportunities and pitfalls of serological diagnosis. Clin Microbiol Infect [periódico online]. 2006;12(6):504-12. Disponível em: https://www.ncbi.nlm.nih.gov/pubmed/16700697. https://doi.org/10.1111/j.1469-0691.2006.01444.x

12. Dupont CD, Christian DA, Hunter CA. Immune response and immunopathology during toxoplasmosis. Semin Immunopathol. [periódico online]. 2012; 34(6):793-813. Disponível em: https://www.ncbi.nlm.nih.gov/pubmed/22955326. https://doi. org/10.1007/s00281-012-0339-3

13. Carvalho AGMA, Lima JS, Lima MSPR, Mota CAX. Diagnóstico laboratorial da toxoplasmose congênita. Rev. Ciênc. Saúde Nova Esperança. 2014;12(1):88-95.

14. Lopes FMR, Gonçalves DD, Mitsuka-Breganó R, Freire RL, Navarro IT. Toxoplasma gondii infection in pregnancy. Braz J Infect Dis [periódico online]. 2007; 11(5):496-506. Disponível em: https://doi.org/10.1590/S1413-86702007000500011

15. Correa D, Cañedo-Solares I, Ortiz-Alegría LB, Caballero-Ortega $\mathrm{H}$, Rico-Torres CP. Congenital and acquired toxoplasmosis: diversity and role of antibodies in different compartments of the host. Parasite Immunol. [periódico online]. 2007; 29(12):651-60. Disponível em: https://www.ncbi.nlm.nih.gov/pubmed/18042171. https://doi.org/10.1111/j.1365-3024.2007.00982.x

16. Furini AAC, Gadotti FC, Bertasso-Borge MS, Pignata RCM, Sanches RMS, Faitarone NC, Santos I, Lima TAM, Cavasini CE, Machado 
RLD. Soroprevalência de Anticorpos anti-Toxoplasma gondii em Amostras de Gestantes no Pré-Natal. Rev. Bras. Cien. Saúde. [periódico online]. 2015. 19(3):199-2014. Disponível em: http:// www.periodicos.ufpb.br/index.php/rbcs/article/view/20576/15070. https://doi.org/10.4034/RBCS.2015.19.03.05

17. Margonato FB, Silva AMR, Soares DA, Amaral DA, Petris AJ. Toxoplasmose na gestação: diagnóstico, tratamento e importância de protocolo clínico. Rev. Bras. Saude Mater. Infant. [periódico online]. 2007; 7(4):381-6. Disponível em: https://doi.org/10.1590/ S1519-38292007000400005

18. Souto BGA, Pereira SMSF. História clínica centrada no sujeito: estratégia para um melhor cuidado em saúde. Arquivos Brasileiros de Ciências da Saúde. [periódico online]. 2011; 36(3):176-81. Disponível em: https://portalnepas.org.br/abcs/article/view/58. https://doi.org/10.7322/abcs.v36i3.58

19. O'Connor A, Doris F, Skirton H. Midwifery care in the UK for older mothers. Br J Midwifery. [periódico online]. 2014; 22(8):568-77. Disponível em: https://doi.org/10.12968/bjom.2014.22.8.568

20. Damasceno SS, Nóbrega VM, Coutinho SED, Reichert APS, Toso BRGO, Collet N. Saúde da criança no Brasil: orientação da rede básica à Atenção Primária à Saúde. Ciênc Saúde Coletiva; [periódico online]. 2016; 21(9):2961-73. Disponível em: https:// doi.org/10.1590/1413-81232015219.25002015

21. Jacquier P, Hohlfeld P, Vorkauf H, Zuber P. Epidemiology of toxoplasmosis in switzerland: national study of seroprevalence monitored in pregnant women 1990-1991. Schweiz. Med Wochenschr. Supp. [periódico online]. 1995; 65:29-38. Disponível em: https://www.ncbi.nlm.nih.gov/pubmed/7716451

22. Findal G, Stray-Pedersen B, Holter EK, Berge T, Jenum PA. Persistent Low Toxoplasma IgG Avidity Is Common in Pregnancy: Experience from Antenatal Testing in Norway. PLOS ONE [periódico online]. 2015; 1-10. Disponível em: https://www.ncbi. nlm.nih.gov/pubmed/26714282. https://doi.org/10.1371/journal. pone. 0145519

23. Amendoeira MRR, Camillo-Coura LF. Uma breve revisão sobre toxoplasmose na gestação. Scientia Medica. 2010; 20(1):113-9. Disponível em: https://dialnet.unirioja.es/servlet/ articulo? codigo $=6268074$

24. Dhakal R, Gajurel K, Pomares C, Talucod J, Press CJ, Montoya JG. Significance of a Positive Toxoplasma Immunoglobulin M Test Result in the United States. J Clin Microbiol [periódico online]. 2015; 53(11):3601-5. Disponível em: https://www.ncbi.nlm.nih. gov/pubmed/26354818. https://doi.org/10.1128/JCM.01663-15

25. Gonçalves R, Urasaki MBM, Merighi MAM, D'Avila CG. Avaliação da efetividade da assistência pré-natal de uma Unidade de Saúde da Família em um município da Grande São Paulo. Rev. Bras. Enferm. [periódico online]. 2008; 61(3):349-53. Disponível em: https://doi.org/10.1590/S0034-71672008000300012

26. Ministério da Saúde (Brasil). Secretaria de Atenção à Saúde.

Departamento de Atenção Básica. Atenção ao pré-natal de baixo risco. Cadernos de Atenção Básica, 32(1):1-320. Brasília: Editora do Ministério da Saúde; 2013.
27. Cantos GA, Prando MD, Siqueira MV, Teixeira RM. Toxoplasmose: Ocorrência de Anticorpos Anti-Toxoplasma gondii e Diagnóstico. Rev. Assoc. Med. Bras. [periódico online]. 2000; 46(4):335-41. Disponível em: https://doi.org/10.1590/S010442302000000400033

28. Mentges BTC, Terezinha I, Rocha M. Apresentações clínicas: atualização da toxoplasmose gestacional. Rev. Panam. Infectol. 2015; 17(1):30-6.

\section{Autores:}

PRISCILA DA HORA NASCIMENTO

Biomédica graduada pela Faculdade Maurício de Nassau, Salvador, BA.

Orcid: https://orcid.org/0000-0002-4227-1744

E-mail: prisciladahora1@gmail.com

Mara Costa CONCEIÇão

Aluna de graduação do Curso de Enfermagem da Universidade do Estado da Bahia (UNEB).

Orcid: https://orcid.org/0000-0003-3194-4402

E-mail: maracostaconceicao@gmail.com

ANa Clara Silva BRANDÃo

Bióloga. Mestre em Ecologia e Biomonitoramento pela Universidade Federal da Bahia (UFBA). Professora do Centro Universitário Maurício de Nassau, Salvador, BA.

Orcid: https://orcid.org/0000-0001-6226-6216

E-mail: clara.bio@gmail.com

Pedro Paulo Oliveira Carneiro

Biomédico. Doutor em Ciências da Saúde pela UFBA. Professor do Centro Universitário Maurício de Nassau, Salvador, BA.

Orcid: https://orcid.org/0000-0001-9600-613X

E-mail: carneiro.ppoc@gmail.com

RúBIA Suely SANTANA Costa

Biomédica. Doutora em Ciências da Saúde pela UFBA. Professora do Centro Universitário Maurício de Nassau, Salvador, BA.

Orcid: https://orcid.org/0000-0002-2663-8833

E-mail: rsuelycosta@gmail.com 